\title{
EEE
}

Homepage: http://publisher.uthm.edu.my/proceeding/index.php/eeee e-ISSN : 2756-8458

\section{Obstacle Detection System for Railways using IoT Sensors}

\author{
Ahmad Akmal Aziz ${ }^{1}$, Khairul Anuar Mohamad ${ }^{1,2 *}$, Afishah \\ Alias $^{3}$ \\ ${ }^{1}$ Faculty of Electrical and Electronic Engineering, \\ Universiti Tun Hussein Onn Malaysia, Parit Raja, 86400, Batu Pahat, Johor, \\ MALAYSIA \\ ${ }^{2}$ Microelectronic and Nanotechnology Shamsuddin Research Center, \\ Universiti Tun Hussein Onn Malaysia, Parit Raja, 86400, Batu Pahat, Johor, \\ MALAYSIA \\ ${ }^{3}$ Faculty of Applied Science and Technology, \\ Universiti Tun Hussein Onn Malaysia, Campus Pagoh, 84600, Pagoh, Johor, \\ MALAYSIA
}

DOI: https://doi.org/10.30880/eeee.2020.01.01.007

Received 02 August 2020; Accepted 06 September 2020; Available online 30 October 2020

\begin{abstract}
Accidents that involve trains on the railway is a crucial issue and need to be avoided. Detecting obstacles is vitally essential nearby and on the railway to ensure the smooth running of the trains. Thus, Keretapi Tanah Melayu Berhad (KTMB) has provided a safety system by relying on visual inspection to monitor the railway. This project aims to provide an alternative precaution measurement that can aid the railway's existing safety system. The project uses two mediums to collect and monitor data about the detecting obstacles nearby and on the railway. The first medium used in this project is the IoT-based system by using FavorIoT as a cloud server platform at the prototype for storing and monitoring data about the distance between obstacles and the prototype. Another medium used is a radar display by using Processing Software to display the distance between obstacles and the device in the range between $0-40 \mathrm{~cm}$. Three ultrasonic sensors are used to provide a dependable notification system to detect the obstacles nearby and on the railway, which placed at the front, right and left sides. Arduino IDE is used to program Arduino NodeMCU, which acts as the brain of the system. The system commands the three ultrasonic sensors to detect the distance between obstacles and the device and send the data via Wifi to the FavorIoT as a cloud server. The prototype managed to collect data in a data stream of FavorIoT and monitor data in the radar display form.
\end{abstract}

Keywords: Arduino, Ultrasonic Sensor, Obstacle Detection, Railway, IoT

\section{Introduction}

Railways are primarily used for intercity passenger transport, and freight transport used together for some urban transport [1]. In Malaysia, there are currently 24 intercity passenger trains running every 
day, consisting of 16 express trains and eight local trains operated by Keretapi Tanah Melayu Berhad (KTMB) to ensure smooth and convenient transportation throughout the metropolitan area [2]. Intercity trains have an average speed of about $50 \mathrm{~km} / \mathrm{h}$, and this can lead to delays in service at peak hours or during the season. Delays and disruption to the railway track because of failures and unplanned maintenance work can have a significant impact on the economy. Frequent delays have caused some problems for KTMB, which can cause significant inconvenience for passengers and reduce the efficiency of the system [3]. There are two main concepts in all transport systems, particularly in the rail sector; safety [4-6] and reliability [7]. Due to the ongoing need to improve rail safety, several European research projects are working on any situation that may pose a threat to rail safety [8]. Particular attention is focused on these circumstances of the existence of objects or obstacles on the railway track [9].

The railway signalling system is the existing system using light signals, axle counters and closedcircuit TV (CCTV) to avoid train collisions, track accidents due to unidentified objects and any unexpected faults or defects to the tracks during train operations [10-13]. In 2012, Uribe et al. proposed a system that capable of warning a driver about possible threats in the route [14]. It employed two complementary approaches which are detecting and tracking the objects using scanning and an algorithm to find candidate hazardous objects, records their trajectories, and forecasts their paths to decide whether a collision course exists. The method has an advantage which is there is a warning signal ahead of time when an object could pose a danger to the safety of the train. Meanwhile, a multi-sensory barrier-consisting of infrared and ultrasonic sensors and a vision system was developed for obstacle detection on railways [15]. The barrier and the vision system analyses the same area continuously. Both systems automatically detect the presence of obstacles in the area, informing the monitoring system about it.

In this work, an obstacles detection system is developed to collect and monitor data about the detecting obstacles nearby and on the railway using IoT-based system and a radar display as an alternative precaution measurement that can aid the railway's existing safety system.

\section{Materials and Methods}

Figure 1 shows the block diagram of an obstacles detection system. There are three ultrasonic sensors in which the ultrasonic sensor 1 with servo motor is located in front of the prototype. In contrast, ultrasonic sensor 2 and ultrasonic sensor 3 are located at both sides of the prototype (right and left). As input of this system, the ultrasonic sensors are acted as detector of distance between obstacles and prototype. The data that have been recorded by the sensors is processed by Arduino software and transferred to the cloud server by using NodeMCU Wi-Fi module and the authorized person can monitor the recorded data at FavorIoT as a IoT platform for this system. The data that have been collected by ultrasonic sensor 1 is also processed by processing software and displayed in form of radar alarm display.

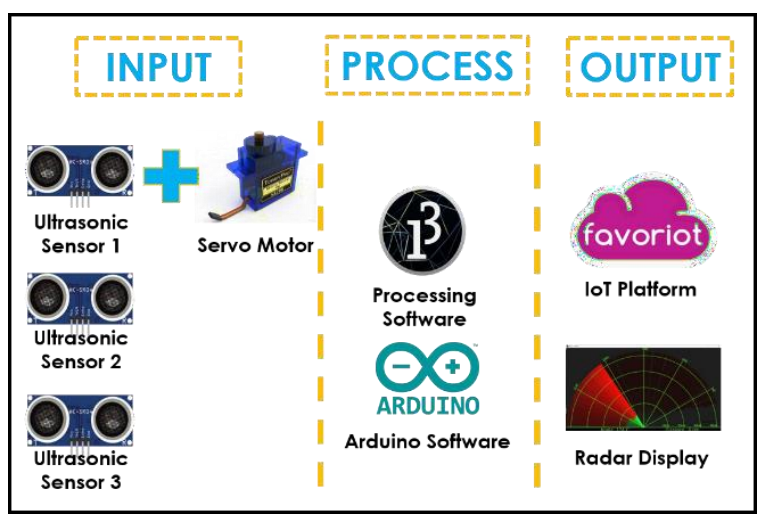

Figure 1: The block diagram of an obstacles detection system 
Figure 2 shows the flowchart of the railway obstacle detection system using IoT technology via FavorIoT platform. The following steps explained the whole working flow principle:

- STEP 1: NodeMCU and the sensors powered up with the batteries as the power supply for this prototype.

- STEP 2: Ultrasonic sensors assemble consist of three ultrasonic sensors are used to detect the distance between the obstacles and the prototype from three locations; in front, right and left of the prototype. Location of ultrasonic sensor 1 with servo motor is in front of the prototype while ultrasonic sensor 2 and ultrasonic sensor 3 located at both sides of the prototype (right and left).

- STEP 3: The data of distance between obstacles and prototype that recorded by the sensors is pushed into cloud server via NodeMCU Wi-Fi module with the help of processor and displayed on the data stream of IoT platform (Favorlot).

- STEP 4: The ultrasonic sensor 1 detects data of distance between obstacles and prototype in the range $\leq 40 \mathrm{~cm}$, the radar display the via processing software, and the prototype is responded and stop the move. If this condition does not happen, the whole process is repeated

- STEP 5: The ultrasonic sensor 2 and 3 detect data of distance between obstacles and prototype in range $\leq 400 \mathrm{~cm}$, the prototype is kept moving. If this condition does not happen, the whole process is repeated.

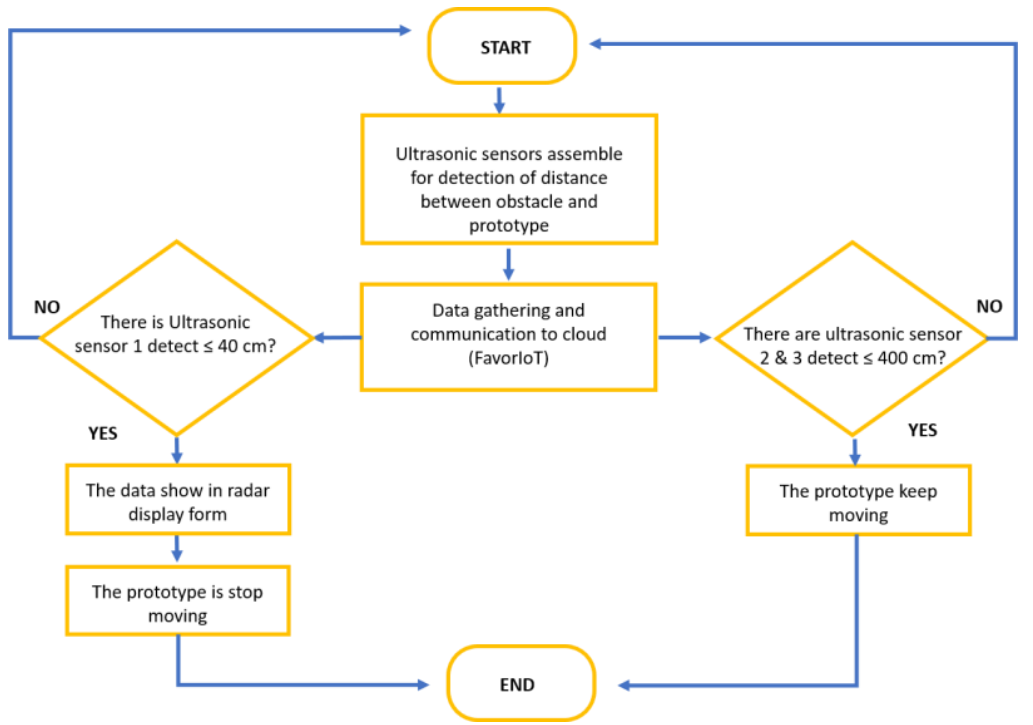

Figure 2: Flowchart the railway obstacle detector system

\section{Results and Discussion}

Figure 3 shows the final prototype of the obstacles detection system. Figure 3(a) shows the circuit connection for three ultrasonic sensors to Arduino NodeMCU with the addition of a motor drive circuit and connection of servo motor to ultrasonic sensor 1. Figure 3(b) shows the final prototype. The performance of three ultrasonics sensor of the obstacles detection system was performed by placing three obstacles at front, left and right side of the prototype, respectively. Table 1 summarizes the results of ultrasonic sensors detection for the distance of $5 \mathrm{~cm}, 10 \mathrm{~cm}$ and $15 \mathrm{~cm}$, respectively. Based on average percentage error, it was observed that accuracy of the ultrasonic sensor is acceptable since the value of percentage errors are less than $7 \%$, and the distance values detect by three ultrasonic sensors are similar range to the actual value. 


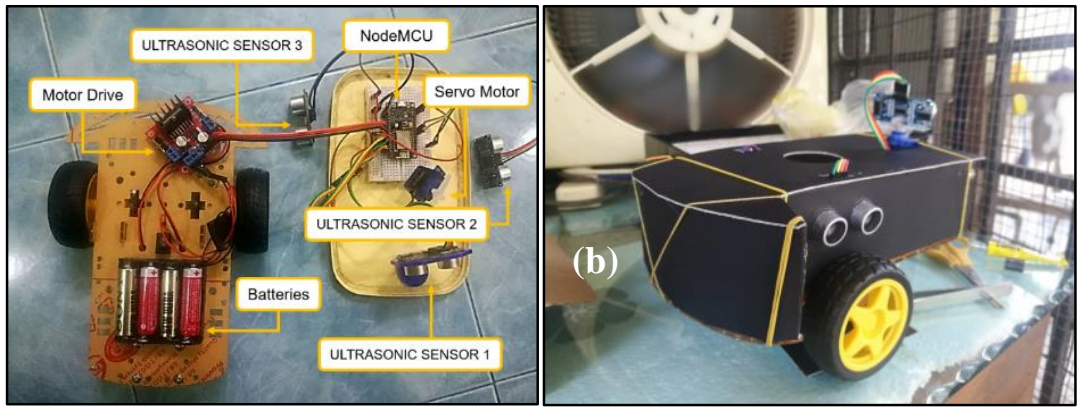

Figure 3: (a) Arrangement of circuit and (b) final prototype

Table 1: Results for ultrasonic sensors performance

\begin{tabular}{cccccc}
\hline \multirow{2}{*}{$\begin{array}{c}\text { Distance } \\
(\mathrm{cm})\end{array}$} & Angle $\left(^{\circ}\right)$ & $\begin{array}{c}\text { Ultrasonic } \\
\text { Sensor 1 }\end{array}$ & $\begin{array}{c}\text { Ultrasonic } \\
\text { Sensor 2 }\end{array}$ & $\begin{array}{c}\text { Ultrasonic } \\
\text { Sensor 3 }\end{array}$ & $\begin{array}{c}\text { Average } \\
\text { Percentage } \\
\text { Error }\end{array}$ \\
\hline \multirow{2}{*}{5} & 89 & 7 & 5 & 5 & \\
& 90 & 6 & 5 & 5 & $6.7 \%$ \\
& 91 & 5 & 5 & 5 & \\
10 & 89 & 9 & 10 & 10 & \\
& 90 & 9 & 10 & 10 & $3.3 \%$ \\
& 91 & 9 & 10 & 10 & \\
& & & & & \\
& 89 & 13 & 15 & 15 & \\
& 90 & 13 & 15 & 15 & $2.9 \%$ \\
& 91 & 15 & 15 & 15 & \\
\hline
\end{tabular}

Figure 4 shows three conditions for testing the obstacles detection system with a radar display for monitoring purpose. The conditions are "no obstacle", "obstacle" and "overlapped obstacles" conditions. Radar display indicates the values for angle distance. Figure 4(a) indicated that there is only green colour indicator existed at the radar display. Green colour indicates there is no object in front of the ultrasonic sensor 1 in the range between $1^{\circ}-180^{\circ}$ and in range distance between $0-40 \mathrm{~cm}$. In this "no obstacle" case, the value of angles is displayed and keep changing depending on the rotation of ultrasonic sensor 1 . At the same time, there is no information on the distance value as no obstacles have existed in front of the prototype. Figure 4(b) exhibited the existence of red colours at the radar display. A red colour indicates that there is an obstacle present at the angle of $168^{\circ}$ with a distance of $20 \mathrm{~cm}$. The location of the obstacles can be determined by referring to the red colour that appears on the radar display. When the radar exhibited a red colour, the value of angle and value of distance are displayed simultaneously at the radar display to inform the exact location of the obstacles. Figure 4(c) also shows indication of red colours appeared on the radar display due to the few obstacles which are overlapped each other. Although there is obstacle detected in the range of the radar, the value of distance does not appear at the radar display due to the multiple distance values recorded at the same angle. The sensor can still detect the obstacles even in overlapped condition. Table 2 shows a summarize of the analysis of radar display for obstacle detection. 

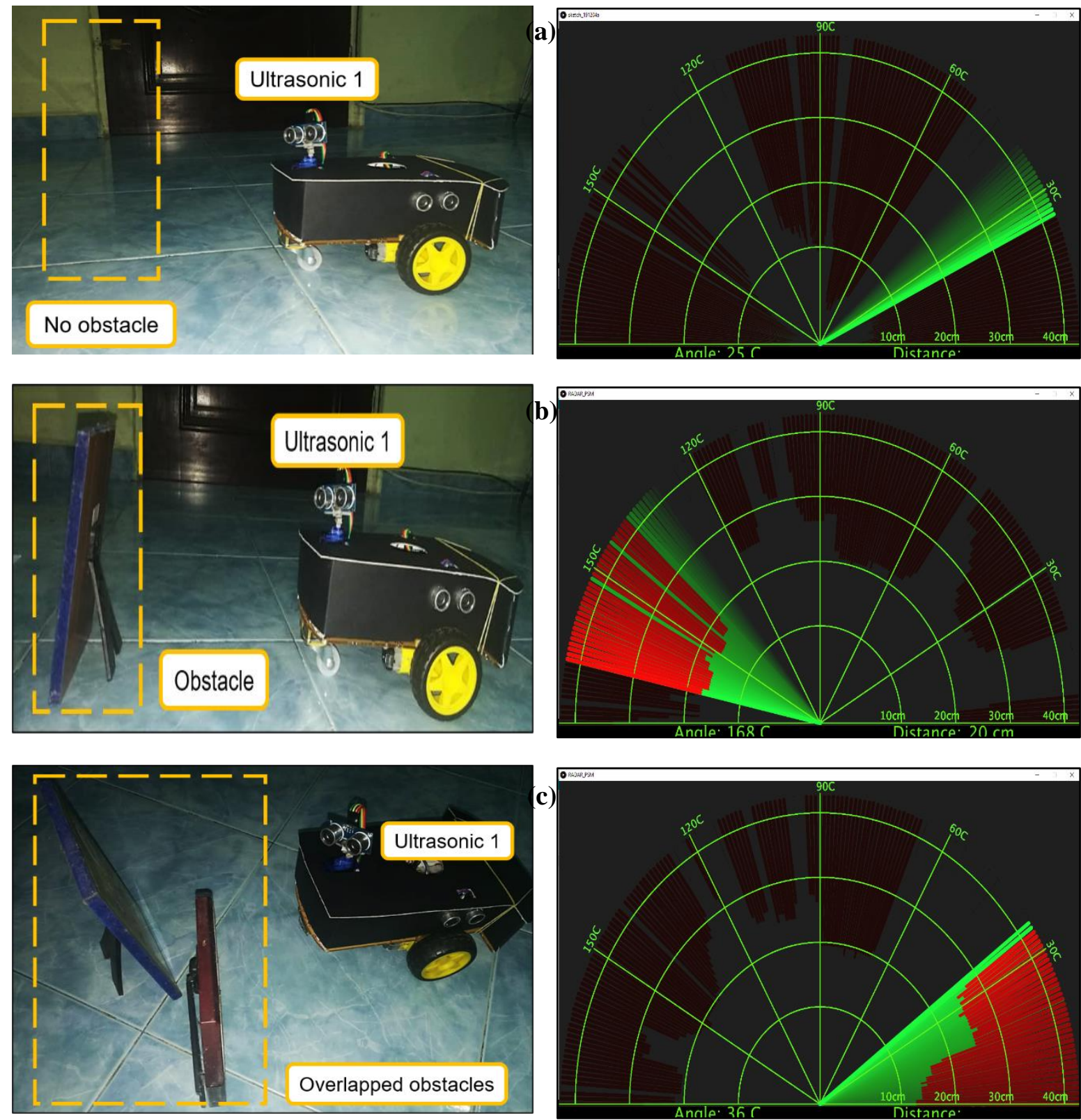

Figure 4: Three testing with (a) "no obstacle", (b) "obstacle" and (c) "overlapped obstacles" conditions with respective radar displays.

Table 3.2: Summary of analysis of radar display for obstacle detection

\begin{tabular}{ccccc}
\hline Condition & $\begin{array}{c}\text { Red Colour } \\
\text { Indicator }\end{array}$ & Value of Angle & Value of Distance & $\begin{array}{c}\text { Location of } \\
\text { Obstacles }\end{array}$ \\
\hline \hline $\begin{array}{c}\text { no obstacle } \\
\text { obstacle }\end{array}$ & No & Yes & No & No existance \\
overlapped \\
obstacles
\end{tabular}

Furthermore, Figure 5 shows the FavorIoT data stream as an IoT based data collection and monitoring system platform. The data stream indicates a serial monitor to display the data. To further analyze the data stream obtained, the data collected can be produced using a graph. Besides, the data can be collected and monitored in further distant by using Internet of Things (IoT) concept. The FavorIoT is an online database which can receive data from IoT end devices and store data using its 
scalable database, manage data stored, secure data securely, and connect other applications or devices to the end-user. The middleware was specially designed to provide a solution for all types of IoT oriented systems such as detection obstacle for railways.

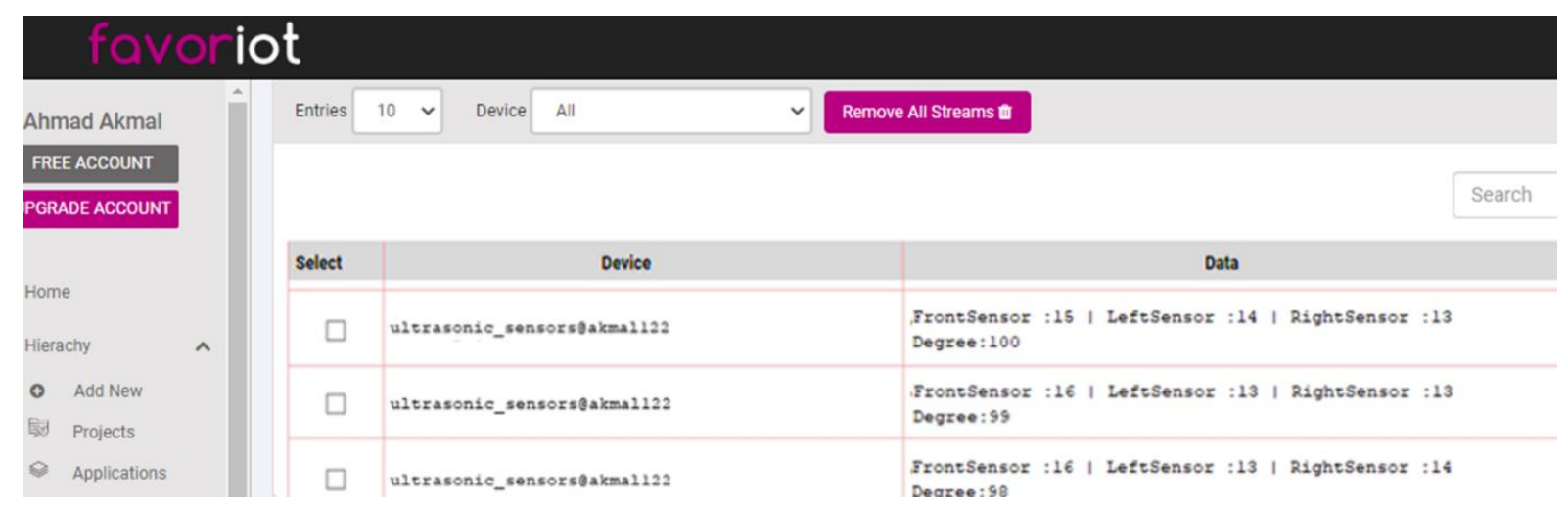

Figure 5: Data collected from obstacle detection system in data stream of FavorIoT

\section{Conclusion}

This work offers an innovative solution for IoT-based obstacles detection at the railway. It is achieved by developing a prototype to run the tasks, and it contained an online database whose middleware is specialized in the Internet of Things. There are two program codes used in this system, which are Arduino IDE and Processing IDE to command an obstacle detector by using three ultrasonic sensors for detecting any obstacles on the railway. Then, the data streams contained in favorloT can help people in charge to monitor any presence of obstacles nearby or on a railway. IoT-based sensors for obstacle detection on railway track provide a solution for giving the exact location of the obstacle.

\section{Acknowledgement}

The authors would like to thank the Faculty of Electrical and Electronic Engineering, Universiti Tun Hussein Onn Malaysia for its support.

\section{References}

[1] M. I. M. Masirin, A. M Salin, A. Zainorabidin, D. Martin, and N. Shamsuddin, "Review on Malaysian Rail Transit Operation and Management System: Issues and Solution in Integration" in IOP Conference Series: Materials Science and Engineering, International Research and Innovation Summit, 6-7 May 2017, Melaka, Malaysia, vol. 226, pp. 012029

[2] A. Roza, S. Koting, and M. R. Karim, "Intercity Land Public Transport Challenges in Developing Country : A Case Study in Peninsular Malaysia," Proc. East. Asia Soc. Transp. Stud., vol. 9, pp. 1-20, 2013

[3] N. S. A. Sukor, M. Subramaniam, and M. I. M. Masirin, "Punctuality of Intercity Trains and Passengers ' Perspective towards Arrival Time Delay,” Res. J. Appl. Sci. Eng. Technol., vol. 5, no. 6, pp. 1998-2002, 2013

[4] J. Reitman, "Achieving Employee Safety - Lessons from the Railroad", IEEE Technology and Society Magazine, Spring 1993

[5] D. Fenner, "Train Protection", IEE Review September 2002

[6] M. Arai, "Railway Safety for the 21st Century", Japan Railway \& Transport Review, no 36, September 2003 
[7] F. J. Restel and M. Zajac, "Reliability Model of the Railway Transporation System with respect to Harzard States", 2015 IEEE International Conference on Industrial Engineering and Engineering Management, 6-9 Dec 2015, Singapore

[8] P. Bon, INRETS; C. Cassir, TU Dresden "Overview of National and European projects European Commission" (railway safety management.) Fifth Framework programme SAMNET thematic Network, February 12th, 2004

[9] M. Ukai, B. T. Nassu and N. Nagamine, "Obstacle Detection on Railway Track by Fusing Radar and Image Sensor", $9^{\text {th }}$ World Congress on Railway Research, 22-26 May 2011, Lille, France

[10] T. Takashige, "Singalling Systems for Safety Railway Transport", Railway Technology Today, Japan Railway \& Transport Review, September 1999

[11] A. Satish, B. Saranya, K. Govardhanaray and D. Palanisamy, "Intelligent System for Automatic Railway Gate Controlling and Obstacle Detection”, Int. J. Sci. Res., vol. 4(8), pp. 24-30, 2017

[12] J. W. Palmer, "The Need for Train Detection", IET Professional Development Course on Railway Signalling and Control Systems 2010 (RSCS2010), 7-11 June 2010, Birmingham, UK

[13] Y. R. Pu, L. W. Chen and S. H. Lee, "Study of Moving Obstacle Detection at Railway Crossing by Machine Vision”, Inf. Technol. J., vol. 13(16), pp. 2611-2618, 2014

[14] J. A. Uribe, L. Fonseca and J. F. Vargas, "Video based System for Railroad Collision Warning", 2012 IEEE International Carnahan Conference on Security Technology (ICCST), 15-18 Oct 2012, Boston, USA

[15] J. J. Garcia-Dominguez, J. Urena-Urena, J. Hernandez-Alonso, M. Mazo-Quintas, J. F. Vazquez and M. J. Diaz, "Multi-Sensory System for Obstacle Detection on Railways", 2008 IEEE Instrumentation and Measurement Technology Conference, 12-15 May 2008, Victoria, Canada 